Transcontinentales

continentales

Sociétés, idéologies, système mondial

Afrique plurielle

\title{
Résiliences et ruptures en Afrique
}

Resilience and change in Africa

\section{Laurent Fourchard}

\section{(2) OpenEdition}

\section{Journals}

Édition électronique

URL : http://journals.openedition.org/transcontinentales/386

DOI : 10.4000/transcontinentales.386

ISBN : 978-2-8218-1408-0

ISSN : 1775-397X

\section{Éditeur}

Editions de la maison des sciences de l'homme

\section{Édition imprimée}

Date de publication : 30 juin 2006

Pagination : 11-20

ISBN : 2200-92169-1

ISSN : 1950-1684

\section{Référence électronique}

Laurent Fourchard, "Résiliences et ruptures en Afrique », Transcontinentales [En ligne], 2 | 2006,

document 2, mis en ligne le 30 septembre 2012, consulté le 08 septembre 2020. URL : http://

journals.openedition.org/transcontinentales/386 ; DOI : https://doi.org/10.4000/transcontinentales. 386

Ce document a été généré automatiquement le 8 septembre 2020

Tous droits réservés 


\title{
Résiliences et ruptures en Afrique
}

\author{
Resilience and change in Africa
}

\author{
Laurent Fourchard
}

1 Les malheurs et les détresses de l'Afrique continuent d'occuper une place prépondérante dans l'agenda politico-médiatique des pays occidentaux : l'effacement d'une partie de la dette et le renforcement de l'aide des pays industrialisés ont même, le temps de l'été 2005, accaparé le paysage médiatique (du très officiel G8 à Gleaneagles à la world charity de Bob Geldof). Au-delà d'une réalité incontestable, que manifestent la marginalisation économique de l'Afrique et la prévalence des guerres, des pandémies et des famines, il convient de se demander si les dernières bonnes nouvelles sur l'Afrique sont strictement conjoncturelles : depuis deux ou trois ans, la croissance économique s'installe, des accords de paix, certes encore fragiles, ont mis un terme au moins provisoire à de nombreux conflits (Angola, Burundi, Congo, Sierra Leone, Liberia), la démocratisation s'enracine dans certains pays alors qu'un esprit de résistance à l'autoritarisme s'affirme dans de nombreux autres. Enfin l'initiative du NEPAD (Nouveau partenariat pour le développement de l'Afrique) voudrait manifester la volonté - au moins rhétorique - d'inverser le cours de l'histoire du continent. En France, plusieurs dossiers et revues témoignent de cette Afrique qui bouge et qui souhaite changer le regard habituel porté sur le continent ${ }^{1}$. S'agit-il d'un afrooptimisme exagéré de certains milieux dirigeants, universitaires ou médiatiques pour mieux cacher les détresses structurelles de l'Afrique, voire pour faire pièce à l'afropessimisme de certains ? ${ }^{2}$ Ou assiste-t-on rien moins qu'à un changement d'époque ?

2 Les ruptures sont pour le moins ardues à qualifier : les ordres politiques, économiques et sociaux obéissent à des temporalités différentes alors qu'une vision englobante du continent est aujourd'hui bien difficile à concevoir. La pluralité des Afriques est une réalité à rappeler sans cesse, et les dynamiques nationales et locales paraissent souvent singulières, comme le souligne ici John Lonsdale et comme viennent le rappeler les études de cas rassemblées dans ce dossier. Ainsi, plutôt que de brosser un bilan de l'Afrique au sud du Sahara, nous avons pris le parti d'identifier quatre questions principales, illustrées dans le dossier par autant d'études de cas, et qui inter-rogent la pertinence des ruptures récentes de l'histoire du continent. Il s'agit du rôle des 
nouveaux acteurs nationaux (Nigeria, Afrique du Sud) et internationaux (Union africaine, NEPAD) sur la scène africaine, des modes de gestion et de règlement des conflits, de l'état de l'avancée de la libéralisation politique et enfin des dynamiques démographiques, migratoires et économiques récentes. Bien évidemment, ces questions ne forment pas synthèse; elles suggèrent plutôt d'insérer l'actualité du continent dans une perspective de plus long terme tout en articulant dynamiques internes et influences extérieures.

\section{La revanche des puissances moyennes en Afrique $?^{3}$}

3 La première rupture apparente renvoie à l'irruption relativement récente de nouveaux acteurs diplomatiques africains sur le continent. L'abolition de l'apartheid et l'élection de Nelson Mandela en Afrique du Sud (1990-1994), la fin du régime dictatorial d'Abacha (1994-1998) et l'élection d'Olusegun Obasanjo au Nigeria (1999) ont permis à ces deux puissances économiques régionales d'acquérir une nouvelle légitimité internationale et de devenir des acteurs incontournables de la scène politique africaine. Cette affirmation des puissances moyennes ne se limite d'ailleurs pas au continent, mais s'inscrit au contraire dans les dynamiques en cours du multi-latéralisme et du régionalisme ${ }^{4}$.

Particulièrement significative est à cet égard l'action diplomatique sud-africaine pour la paix en Afrique dont Garth le Pere nous livre ici une synthèse éclairante. La nouvelle politique étrangère sud-africaine renferme une dimension panafricaine qui relie inextricablement le destin du pays à l'avenir du continent africain. La promotion de la démocratie et des droits de l'homme a semble-t-il laissé davantage la place à la résolution des conflits et à la promotion de la paix, politiques centrales dans la Renaissance africaine promue par le président Thabo Mbeki depuis 1999. Bien que cet activisme diplomatique se fasse dans un cadre de plus en plus multilatéral, il ne se fait pas toujours sans heurt: il est parfois contesté par les partenaires africains et occidentaux (trop interventionniste en Côte d'Ivoire pour la France, trop peu au Zimbabwe pour la Grande-Bretagne). Il accompagne par ailleurs la conversion tardive de l'African National Congress (ANC) aux impératifs de l'orthodoxie néo-libérale et la pénétration des intérêts économiques sud-africains en Afrique.

5 Ainsi se pose la question de l'hégémonie de ce pays sur le continent. Trois échelles d'analyse sont à dissocier ${ }^{5}$. 1) La SACU (Southern African Customs Union), union douanière vieille de plus d'un siècle entre l'Afrique du Sud, le Botswana, le Lesotho, la Namibie et le Swaziland, représente sans doute l'expression la plus institutionnalisée de cette hégémonie régionale ; celle-ci ne doit cependant pas être surestimée ${ }^{6}$. 2) Au sein de la SADC (Southern African Development Community), principale organisation régionale qui comprend 14 pays de la région, cette domination est contestée. 3) Enfin au nord du Zambèze, cette hégémonie passe par une avancée simultanée des capitaux et de la diplomatie sud-africaine. L'Afrique du Sud est ainsi leader des pays africains dans les investissements sur le continent ; elle n'a cependant pas encore détrôné les puissances occidentales en termes d'investissements ${ }^{7}$. Son hégémonie est incomplète et contestée par d'autres États d'Afrique, notamment par le Nigeria.

6 Pays de 130 à 150 millions d'habitants, premier producteur de pétrole en Afrique subsaharienne, le Nigeria est revenu en force dans la diplomatie du continent depuis 1999 (médiation dans plusieurs conflits dont le Soudan, missions de maintien de la paix 
en Afrique de l'Ouest). Sa rente pétrolière et ses réserves de gaz lui donnent les moyens de sa diplomatie et en font un partenaire obligé des États-Unis et des autres pays occidentaux. Ses nombreux conflits internes, l'inefficience et la corruption de l'État, la faiblesse de sa monnaie, l'effondrement de son système scolaire et sanitaire et la méfiance que suscitent les investisseurs privés nigérians à l'étranger limitent néanmoins ses prétentions internationales.

7 L'Afrique du Sud, plus encore que le Nigeria, a joué un rôle central dans la création de l'Union africaine et du NEPAD. Alors que les débats étaient depuis l'indépendance dominés par les projets d'intégration régionale, l'UA et le NEPAD représentent un espoir d'une union continentale réunissant pour la première fois toute l'Afrique $d u$ Nord au Sud ${ }^{8}$. Le concept, simple, repose sur l'idée que les pays en développement ne peuvent espérer attirer l'argent des pays riches sans leur en garantir une utilisation judicieuse par un mécanisme d'évaluation des régimes africains par leurs pairs. Dans l'immédiat cependant, le NEPAD doit encore faire ses preuves et emporter l'adhésion de ses partenaires africains, ce qui est loin d'être acquis'. Ses plus grandes réussites sont d'avoir suscité une réflexion sur le volume de l'aide et d'avoir intégré des fonctions de maintien de la paix qui n'étaient pas prévues à l'origine ${ }^{10}$.

\section{Conflits, prévention et règlements}

La fin de la guerre froide aurait-elle changé la nature des conflits en Afrique ? Certains analystes ont soutenu qu'à des conflits idéologiques opposant une armée régulière et une guérilla soutenue par des parrains internationaux pendant la guerre froide auraient succédé des new wars, des conflits ethniques et/ou économiques entre une multitude d'acteurs désorganisés avec une forte victimisation des civils ${ }^{11}$. Comme le signale Vincent Foucher, s'il y a une part de vérité dans cette analyse, la rupture n'est pas aussi radicale qu'on le pense car le phénomène des enfants soldats ou les clivages ethno-politiques internes étaient déjà présents pendant la guerre froide.

Ainsi en est-il du conflit au Soudan qui oppose le Sud au Nord depuis les années 1950. Gérard Prunier montre bien qu'en dépit de la signature des accords de paix en 2005, la marche vers la paix est largement compromise par l'apparition d'une nouvelle zone d'affrontement au « Darfour» (2003-2004) exclue des accords de paix, et par la mise en exploitation depuis 2002 des champs pétrolifères du Sud qui aiguise les appétits des anciens ennemis. Alors que de multiples conflits partiels entravent l'espoir d'une paix globale, la demande d'indépendance réclamée par le Sud depuis 1955 et prévue dans les accords de paix sera au cœur des oppositions à venir. Les États-Unis regardent de très près l'évolution de la situation, le Soudan représentant pour eux un pays à haut risque en Afrique.

De fait, les attentats du 11 septembre 2001 ont considérablement modifié la donne en propulsant la «sécurité » au rang des priorités mondiales. La communauté internationale a aujourd'hui établi une équivalence entre sécurité et développement, comme dix ans auparavant elle avait mis sur le même plan démocratie, bonne gouvernance et développement ${ }^{12}$. L'Afrique suscite donc un relatif nouvel intérêt alors que la communauté internationale avait paru se désintéresser du continent à la suite des désastreuses opérations humanitaires en Somalie (1991) et au Rwanda (1994). C'est notamment le cas pour les Nations unies et pour les principales puissances occidentales (États-Unis, Grande-Bretagne, France). Ainsi les mandats des nouvelles opérations de 
l'ONU sont-ils plus larges, les forces de paix plus agressives et plus nombreuses (48 000 hommes en août 2004, soit deux tiers des effectifs des Casques bleus dans le monde) ${ }^{13}$. L'implication américaine est également différente aujourd'hui. Les ÉtatsUnis perçoivent effectivement en Afrique un double danger, celui du terrorisme et de l'interruption de la fourniture de pétrole, perception nouvelle née en 2002 au sein du Pentagone et du Département d'État ${ }^{14}$. Les dépenses militaires américaines sur le continent ont ainsi doublé entre 2002 et 2005 (597 millions de dollars) par rapport à la période 1998-2001, représentant un tiers du total du budget des trois décennies de la guerre froide (1,5 milliard de dollars). Par ailleurs, les sociétés privées américaines utilisent aujourd'hui une «stratégie philanthropique » leur permettant de nouer des relations directes avec les communautés locales sur place. Les objectifs et la portée de ces actions, ainsi que l'idéologie sous-jacente, sont nouveaux et bien différents de ceux adoptés lors de la guerre froide ${ }^{15}$. Pour les puissances européennes, en revanche, l'Afrique peut apparaitre aujourd'hui comme un «espace de valorisation diplomatique » ou "d'un nouvel interventionnisme libéral ${ }^{16}$. La mise sur pied de la Commission pour l'Afrique par Tony Blair et l'inscription de l'Afrique comme priorité de l'année 2005 du G8 peuvent paraître comme un nécessaire investissement du parti travailliste auprès de partenaires africains - notamment de l'Afrique du Sud - qui n'ont guère apprécié l'intervention britannique en Irak. L'intervention française en Iturie (République démocratique du Congo) est aussi un coup multilatéraliste en réponse à l'unilatéralisme américain ${ }^{17}$. Reste que les champs traditionnels d'intervention des deux anciennes puissances coloniales demeurent fortement marqués pas des actions bilatérales comme en témoigne l'engagement militaire de la France en Côte d'Ivoire (depuis 2002) ou britannique en Sierra Leone (depuis 2000). La tutelle exercée sur ces pays par les anciennes métropoles pour construire la paix traduit bien ce télescopage du temps long (l'héritage colonial) et du temps court (un interventionnisme légitimé a posteriori par l'ONU) ${ }^{18}$.

\section{Libéralisation politique et résilience de l'autoritarisme}

11 Après trois décennies de guerre froide marquée par la prévalence des régimes autoritaires, la décennie 1990 en Afrique s'inscrit dans un processus de libéralisation politique initié par les premières conférences nationales souveraines qui ont autorisé le multipartisme et les médias indépendants, libéré la parole politique et procédé à la mise en place de nouvelles constitutions. Ce processus s'inscrit dans ce qu'il est convenu d'appeler la «troisième vague » démocratique, limitée aux pays de l'Europe méditerranéenne aux lendemains de la Révolution des Æillets (1974), puis élargie depuis deux décennies à l'Europe de l'Est, à l'Amérique latine et à l'Afrique subsaharienne ${ }^{19}$. Ces démocraties demeurent partout fragiles en Afrique où le terme même pose question. Dans la plupart des cas, il y eut bien souvent conversion de l'ancien personnel politique des périodes autoritaires aux vertus de la démocratie représentative avec tous les travers désormais bien connus: campagnes électorales truquées, forte marchandisation du vote, liberté de presse rarement garantie, recours à la torture, à l'intimidation et à l'élimination physique des leaders d'opposition ${ }^{20}$. Avec le discours ambiant sur la «bonne gouvernance ", les habillages démocratiques ont pris davantage d'importance comme en témoignent par exemple les «révisions constitutionnelles » autorisant les présidents à modifier en leur faveur le jeu électoral ${ }^{21}$. L'inflation des termes pour tenter de définir ces régimes (autoritarisme électoral, 
démocratie autoritaire, régimes semi-autoritaires, semi-démocratiques, démocratiques en survie, hybrides) témoigne des difficultés de catégorisation. Elle manifeste aussi une résilience de l'autoritarisme hérité des périodes coloniales et post-coloniales, sans parler des restaurations autoritaires qui ont suivi la première vague de libéralisation (Niger, Congo Brazzaville, Burundi) et des pays imperméables à tout changement mais soutenus à bout de bras par des pays amis (Togo, Gabon, Zimbabwe...). En 2000, Christophe Jaffrelot constatait que la "troisième vague" était étale, quand elle n'amorçait pas un reflux ${ }^{22}$. En 2006, le nombre de pays d'Afrique où l'alternance politique est possible est à peine plus important qu'en $2000^{23}$, alternance qui par ailleurs ne suffit pas à garantir les dérives autoritaires, y compris dans les pays considérés - sans doute abusivement - à «tradition démocratique » comme le Sénégal et le Botswana ${ }^{24}$.

12 L'esprit de résistance démocratique s'enracine néanmoins progressivement dans la plupart des pays, parallèlement au maintien des comportements de dérision politique dont ont bien rendu compte les travaux de "politique par le bas» initiés dans les années $1980^{25}$. En témoigne la contribution de Brice Rambaud sur la presse togolaise sous le régime Eyadema (1967-2005). Celle-ci comptait en 2003 quelque 43 titres, faisant preuve d'une vitalité remarquable compte tenu de l'autoritarisme du régime. Derrière l'apparente diversité se cache néanmoins une homogénéité certaine: il s'agit d'une presse d'opinion, encore très liée aux partis politiques d'après l'auteur. Par ailleurs, la médiatisation de la parole politique s'inscrit dans une certaine continuité. À la langue de bois du parti unique succèdent les commentaires de la presse aux ordres, tandis que le vocabulaire de la dérision autrefois clandestin ${ }^{26}$ se dévoile désormais dans les journaux de l'opposition par un recours fréquent aux métaphores et à la théâtralisation du politique. Les journaux d'opposition n'en demeurent pas moins fragiles, soumis à de multiples pressions politiques et administratives, limités par un tirage et une diffusion faibles et pratiquant encore l'autocensure pour éviter la sanction ou la répression.

Plus généralement, dans les pays dominés par des régimes sans alternance (modèle dominant aujourd'hui sur le continent), il faut bien reconnaitre que les partis d'opposition sont faibles, souvent financés sur les deniers personnels de leur leader, soumis aux tracas administratifs ou au harcèlement du pouvoir en place, faiblement implantés dans les campagnes et surreprésentés dans les capitales. Mais contrairement à ce que l'on imagine, beaucoup ont une tradition longue (mais souvent interrompue) de lutte politique. Ainsi, de nombreux partis actuels de l'opposition ont une filiation avec les partis qui ont émergé pendant la courte période de libéralisation politique des empires français et britanniques (années 1940 et 1950) ${ }^{27}$. À cet égard, les relations entre le pouvoir, les partis et la presse d'opposition actuellement à l'œuvre ne sont pas sans rappeler cette phase d'initiation à la vie politique et associative (premiers partis de masse, premières assemblées territoriales, premières élections générales) qui a caractérisé la fin des grands empires coloniaux même si le contexte économique et social s'est évidemment modifié depuis.

\section{De nouvelles dynamiques démographiques, économiques et migratoires?}

14 En cinquante ans, et malgré la mortalité des grandes pandémies, on est passé d'une Afrique vide où la pénurie de main d'œuvre constituait le problème central des 
administrations et des entreprises à une Afrique marquée par le chômage de masse, le sous-emploi et le travail informel ${ }^{28}$. Rappelons que la population de l'Afrique subsaharienne est passée de 160 millions en 1940 à 700 millions en 2000 et devrait atteindre 1,3 milliard d'habitants en 2025, soit l'augmentation la plus rapide jamais enregistrée dans l'histoire du monde. La transition démographique a été plus tardive et plus rapide qu'ailleurs, mais elle entre depuis les années 1990 dans la seconde phase, celle du ralentissement de la croissance sous l'effet de la baisse de l'indice de fécondité (passé de 6,7 enfants par femme en 1985 à 5,4 en 2002) ${ }^{29}$. Cette croissance démographique est aujourd'hui ralentie par la pandémie du sida : sur les 40 millions de personnes atteintes dans le monde 25 millions sont des Africains. La prise de conscience de l'ampleur du désastre a été bien tardive ${ }^{30}$.

Le marché du travail s'est atrophié davantage avec la crise économique des années 1980 et 1990, la réduction des dépenses publiques et la fermeture des -frontières en Europe aux migrations des pays du Sud. Les traditions historiques de mobilité, de commerce et de migrations à longue distance ont néanmoins facilité une adaptation constante aux changements politiques et économiques. Ainsi, l'accé-lération de l'extraversion du continent au XXesiècle a remodelé la carte des densités de population en faveur de "l'Afrique utile et urbaine" (villes côtières, zones minières, zones d'agriculture d'exportation). Il existe aussi depuis fort longtemps une circulation migratoire intense à l'échelle régionale entre des pôles d'oppor-tunités économiques (Kenya, Nigeria, Côte d'Ivoire, régions minières du Congo, Afrique du Sud) et des voisins plus défavorisés. À cet égard, le processus de régionalisation passe en Afrique sur l'exploitation de la frontière et du commerce transfrontalier qui n'obéit évidemment pas au découpage des organisations régionales ${ }^{31}$.

16 Depuis une quinzaine d'années, on assiste par ailleurs à une diversification des champs migratoires. Il y a eu, probablement depuis le début des années 1990, à la fois une réactivation des routes de l'immigration saharienne et atlantique en direction de l'Afrique du Nord et de l'Europe occidentale, et une diversification des courants migratoires vers de nouvelles destinations (Turquie, Europe de l'Est, Moyen-Orient, Afrique australe) même si aucun chiffre fiable ne peut réellement être avancé ${ }^{32}$. Il y a bien entendu côté européen, une surmédiatisation de la «menace" migratoire africaine ${ }^{33}$. Il n'en reste pas moins que le gradient migratoire est considérable sur les enclaves espagnoles du Maroc (Ceuta, Mellila) et sur les îles européennes des côtes de l'Afrique (Lampedusa entre la Sicile et la Libye, les Canaries) ${ }^{34}$. Cette pression a certainement été renforcée, depuis le milieu des années 1990, par la fermeture progressive de la Côte d'Ivoire aux travailleurs étrangers, notamment d'Afrique de l'Ouest francophone. L'ouverture simultanée de l'Afrique du Sud en 1990-1994 et, dans une moindre mesure, du Botswana et de la Namibie a provoqué, selon Aurelia Wa Kabwe-Segatti, un appel d'air considérable pour les travailleurs des pays voisins (Mozambique, Lesotho, Swaziland, Mozambique) mais aussi - et c'est plus nouveau pour ceux de l'ensemble du continent. Comme le montre bien l'auteur, les gouvernements de ces trois pays revendiquent un cosmopolitisme et un panafricanisme tout en appliquant des politiques plutôt restrictives pour l'accès à un marché de l'emploi anémié. Ainsi l'Afrique du Sud a-t-elle reconduit à la frontière pas moins de 1,6 million de personnes entre 1993 et 2004, tout en élaborant depuis 2002 une politique de quotas pour des «migrants compétent ». Aujourd'hui plus encore qu'au début des 
années 1990, la pression migratoire est donc très forte aux deux extrémités du continent.

\section{L'Afrique et la mondialisation}

17 La période coloniale a fortement accentué l'extraversion économique de l'Afrique qui est devenue dès lors très sensible aux fluctuations de l'économie mondiale. Elle a bénéficié de la croissance des Trente Glorieuses, mais a plus encore été affectée par la crise des années 1930 et par celle des années 1970-1980 ${ }^{35}$. Dans ces conditions, il est trop tôt pour dire si la croissance de $5 \%$ l'an qui s'observe depuis quelques années est le signe avant-coureur d'un changement de cycle pour le continent. Avec 34 PMA (pays les moins avancés) sur un total de 49 et 34 PPTE (pays pauvres très endettés) sur 42 l'Afrique demeure économiquement marginalisée. Décidée en juin 2005, la suppression de la dette de 18 pays pauvres, dont 14 pays en Afrique subsaharienne, constitue certainement un changement notable dans l'approche qui avait prévalu depuis les années 1970 dans la gestion de la dette. Mais ces dernières évolutions ne suffiront pas à sortir le continent du sous-développement. L'Afrique demeure confrontée à plusieurs problèmes macro-économiques majeurs qui remontent à la période coloniale, tels que la subordination quasi exclusive à l'égard des exportations des produits de base, l'absence de tissu industriel, le faible taux d'épargne et d'investissement, la couverture limitée des besoins de santé et d'éducation ${ }^{36}$. L'Afrique du NEPAD, comme l'Afrique coloniale, n'a pas réussi à attirer les investissements directs étrangers (IDE) en dehors des secteurs miniers et pétroliers traditionnels, investissements qui en tout état de cause ne suffiront certainement pas à enclencher un processus économique vertueux ${ }^{37}$. L'économiste Jeffrey Sachs estime ainsi que l'Afrique est trop pauvre pour décoller sans un soutien international massif ${ }^{38}$.

Depuis quelques années, la communauté internationale réclame de manière récurrente un renforcement de l'aide publique au développement (ADP) même si celle-ci ne cesse de susciter des réserves y compris au sein de la Banque mondiale ou du FMI ${ }^{39}$. Bien entendu le constat d'échec ne date pas d'aujourd'hui. L'histoire des quarante dernières années a montré l'inadéquation des modèles importés, qu'ils soient, selon les périodes, liés à l'idéologie participationniste des animateurs ruraux, au schéma marxisteléniniste des planificateurs, aux opérations de développement des ingénieurs ou, plus récemment, aux thérapies libérales ${ }^{40}$. On pourrait y ajouter les politiques de réduction de la pauvreté de ces dernières années, notamment les Objectifs de développement du millénaire (OMD) adoptés par l'Assemblée générale de l'ONU en septembre 2000, ultime avatar des politiques d'ajustements structurels pour certains ${ }^{41}$, récit positif et positiviste occidental oblitérant totalement les questions politiques pour d'autres ${ }^{42}$. La lutte contre la pauvreté constitue ainsi le dernier catéchisme de la communauté internationale. À l'instar des autres discours du développement, il est une «machine anti-politique » ou une "machine à dépolitiser $"{ }^{43}$. De fait, ce combat fait la quasiunanimité des États, des appareils médiatiques et politiques de gauche comme de droite en Occident; il refuse de considérer les contextes politiques locaux et nationaux des pays récipiendaires ${ }^{44}$.

19 In fine, on en revient encore à l'État et aux institutions capables de mettre en œuvre des politiques publiques. Malgré un legs colonial commun, les États en Afrique disposent de traditions bien différentes à l'échelle du continent : quoi de commun entre 
l'Afrique du Sud, héritière d'un puissant appareil d'État capable de mener une politique intérieure et internationale ambitieuse, le Nigeria qui compte bien peu sur l'Aide publique au développement pour afficher ses ambitions, et la majorité des États dépendants de l'aide internationale dont les marges de manœuvre sont réduites à l'extrême? Cependant, même dans les pays les plus pauvres, le rôle que joue l'État fût-il embryonnaire - reste à bien des égards déterminant sur la moyenne durée. À la différence des administrations coloniales, les gouvernements des indépendances ont massivement investi dans les infrastructures sanitaires et scolaires et ce jusqu'à aujourd'hui ${ }^{45}$. Il faut ainsi relativiser le déclin irrémédiable de l'État en Afrique (et ailleurs) sous l'effet de la mondialisation ${ }^{46}$. Bien plus qu'à une privatisation avancée de l'État, on assisterait à sa recomposition permanente par une délégation (ou « décharge ») de ses fonctions de souveraineté et de coercition à des agents privés, délégations qui furent également communes aux formations étatiques précoloniales et aux États coloniaux et postcoloniaux ${ }^{47}$. John Lonsdale rappelle, à propos du Kenya, que l'État fonctionne en Afrique. Il ajoute cependant que la forte insécurité foncière et la fragilité des structures de l'État héritées de la période coloniale demeurent des éléments déterminants dans le différentiel de développement avec l'Asie. C'est rappeler une fois encore les contingences politiques dans les politiques de développement.

\section{NOTES}

1. "Vues d'Afrique ", Esprit, $\mathrm{n}^{\circ} 317$, août-septembre 2005 ; «L'Afrique qui marche. Loin du Darfour et de la Côte d'Ivoire, les réussites d'un continent », Courrier International, $\mathrm{n}^{\circ} 762$, juin 2005 ; Sylvie Brunel, L’Afrique dans la mondialisation, Paris, La Documentation française, $\mathrm{n}^{\circ} 8048$, 2005.

2. Stephen Smith, Négrologies, Pourquoi l'Afrique meurt, Paris, Calmann-Lévy, 2003.

3. Bertrand Badie, «La revanche des puissances moyennes ? ", in L'État du Monde 2006, Paris, La Découverte, 2006, pp. 26-30.

4. Ibid.

5. Chris Alden et Mills Soko, "South Africa's Economic Relations with Africa: Hegemony and its Disscontents ", Journal of Modern African Studies, 43, 3, 2005, pp. 367-392. Voir aussi l'analyse plus précise de Philippe Gervais-Lambony, L'Afrique du Sud et les États voisins, Paris, Armand Colin, 1997.

6. Dominique Dabon (éd.), L'après-Mandela. Enjeux sud-africains et régionaux, Paris, Karthala/ MSHA, 1999, pp. 7-8.

7. Sur la période 1996-2000, les investissements privés directs restent dominés par les États-Unis (37\%), la France (18\%) et de la Grande-Bretagne (13\%). La progression de l'Afrique du Sud est cependant beaucoup plus rapide que celle des partenaires occidentaux.

8. Jean Coussy, «Les utopies servent-elles l'intégration africaine?», in Dario Chi (coor.), Les défis de l'Afrique, Paris, Dalloz, 2006, pp. 137-141

9. En juillet 2005, le NEPAD rassemblait la moitié des États africains. Le président sénégalais Abdoulaye Wade rejetait la responsabilité de l'inaction de l'organisation sur « le secrétariat du NEPAD et de ses bureaucrates ", installés en Afrique du Sud. Jean-Pierre Turquoi, « Le monde se mobilise pour faire sortir l'Afrique de la pauvreté », Le Monde, mercredi 6 juillet 2005, p. 2. 
10. J. Coussy, art. cit., p. 140-141.

11. Vincent Foucher, « Conflits d'Afrique, conflits globaux », in Dario Chi, op. cit, p. 119.

12. Roland Marchal et Richard Banégas, "Interventions et interventionnisme en Afrique ", Politique Africaine, $\mathrm{n}^{\circ}$ 98, juin 2005, p. 17.

13. V. Foucher, art. cit., pp. 124-126.

14. Sandra Barnes, "Global Flows: Terror, Oil and Strategic Philanthropy », African Studies Review, vol. 48, n 1, avril 2005, pp. 1-22.

15. Ibid, p. 1.

16. V. Foucher, art. cit, p. 127 ; voir aussi le numéro 98 de Politique Africaine : « Pax Africana? Le nouvel interventionnisme libéral », juin 2005.

17. R. Marchal et R. Banégas, art. cit, p. 11.

18. Ruth Marshall, «La France en Côte d'Ivoire : l'interventionnisme à l'épreuve des faits » et Aline Leboeuf, "La réforme britannique du secteur de la sécurité en Sierra Leone. Vers un nouveau paradigme? » Politique Africaine, $n^{\circ} 98$, juin 2005, pp. 21-78.

19. Samuel P. Hutington, "Democracy's Third Wave », The Journal of Democracy, vol. 2, $\mathrm{n}^{\circ} 2$, 1991. Patrick Quantin, «La difficile consolidation des transitions démocratiques africaines des années 1990 », in Christophe Jaffrelot (dir.), Démocraties d'ailleurs. Démocraties et démocratisations hors d'Occident, Paris, Karthala, 2000, pp. 479-508.

20. Dans une abondante littérature voir par exemple en l'Afrique de l'Ouest francophone, Richard Banégas, La démocratie «à pas de caméléon ». Transition et imaginaires politiques au Bénin, Paris, Karthala, 2003 ; Mamadou Gazibo, Les paradoxes de la démocratisation en Afrique. Analyse institutionnelle et stratégique, Québec, Presses de l'Université de Montréal, 2005.

21. Le Burkina de Compaore, l'Ouganda de Museveni, le Botswana de Mogae et peut être demain le Nigeria d'Obasanjo.

22. Christophe Jaffrelot (dir.), Démocraties d'ailleurs. Démocraties et démocratisations hors d'Occident, Paris, Karthala, 2000.

23. Bénin, Cap-Vert, Ghana, Kenya, Malawi, Mali, Maurice, Sao Tome, Sénégal, Zambie. L'Afrique $\mathrm{du}$ Sud, le Botswana et la Namibie sont des exemples de démocratie consolidée (plusieurs élections libres successives) sans alternance politique.

24. Vincent Foucher et Tarik Dahou, "L'alternance au Sénégal », Politique Africaine, n 96, décembre 2004, pp.5-22 ; Ian Taylor, «Le Botswana face à la montée de l'autoritarisme", Politique Africaine, $\mathrm{n}^{\circ}$ 99, octobre 2005, pp. 116-129.

25. Jean-François Bayart, Achille Mbembe et Comi Toulabor, Le politique par le bas en Afrique noire. Contributions à une problématique de la démocratie, Paris, Kathala, 1992.

26. C. Toulabor, «Jeux de mots, jeux de vilains. Lexique de la dérision politique au Togo », ibid., p. 111.

27. Ghana, Nigeria, Togo, Cameroun, Burkina Faso, Kenya. La liste n'est pas exhaustive.

28. Frederick Cooper, Decolonization and African Society. The Labor Question in French and British Africa, Cambridge, Cambridge University Press, 1995. Le chômage urbain comme le secteur informel citadin sont cependant bien antérieurs aux années 1940. John Iliffe, The African Poor. A History, Cambridge, Cambridge University Press, 1987.

29. Tamany Safir, «Les défis de l'Afrique en matière de population à l'horizon 2020 ", in Dario Chi, op. cit., p. 54.

30. En Afrique du Sud, il s'agit peut-être de l'un des plus grands échecs du gouvernement démocratique. Alan Jeeves, "Assessing a Decade of Democracy in Southern Africa ", Revue canadienne des études africaines, 38, 3, 2004, p. 516.

31. Daniel C. Bach, «Regionalism and Globalisation in Sub-Saharan Africa: Revisiting a Paradigm », in D. C. Bach (éd.), Regionalisation in Africa : Integration and Disintegration, Oxford, James Currey, 1999 ; Anthony Asiwaju (éd.), Partitioned Africans : Ethnic Relations Across Africa's 
International Boundaries, 1884-1984, Lagos, University of Lagos Press, 1984 ; Janet Roitman, « The Garrison-Entrepôt », Cahiers d'études africaines, 150-152, 1998, pp. 297-329.

32. Sylvie Bredeloup et Olivier Pliez, "Migrations entre les deux rives du Sahara ", Autrepart, Revue de sciences sociales au Sud, n³6, 2005, pp. 3-20 ; Emmanuelle Grégoire et Jean Schmitz, "Afrique noire et monde arabe ", Autrepart, n 16, 2000 ; A. Bouillon (dir.), Immigration africaine en Afrique du Sud, Les migrants francophones des années 1990, Paris, IFAS/Karthala, 1999; Laurence Marfaing et Steffen Wippel (éds.), Les relations transsahariennes à l'époque contemporaine : un espace en constante mutation, Paris, Karthala/ZMO, 2003.

33. S. Bredeloup et O. Pliez, art. cit., p. 5.

34. On parle de gradient migratoire pour évoquer le différentiel de développement économique et démographique combiné à la proximité géographique entre deux zones données.

35. $\mathrm{k}$ Cooper, Africa since 1940. The Past of the Present, Cambridge, Cambridge University Press, 2002, pp. 91-132. Hélène d'Almeida-Topor et Monique Lakroum, L'Europe et l'Afrique. Un siècle d'échanges économiques, Paris, Armand Colin, 1994.

36. Philippe Hugon, L'économie de l'Afrique, Paris, La Découverte, 2000, p. 20.

37. J. Coussy, art. cit, p. 110 ; Cooper, op. cit, pp. 99-102. Voir le dernier rapport de la CNUCED sur les IDE : Le développement économique en Afrique. Repenser le rôle de l'investissement étranger direct, Rapport 2005 de la Conférence des Nations unies sur le commerce et le développement. http://www. unctad.org/Templates.

38. http://www.unmillenniumproject.org/reports. Voir aussi J. Sachs, The End of Poverty, New York, Penguin Books, 2005.

39. Ces deux institutions ont récemment mis à mal les effets de l'aide internationale sur les pays récipiendaires. Laurence Caramel, «Afrique : au bon cœur du G8 », Le Monde, mardi 5 juillet 2005.

40. P. Hugon, op. cit., p. 108.

41. Tunde Zack Williams et Giles Mohan, «Africa from SAPS to PRSP : plus ça change, plus c'est la même chose", Review of African Political Economy, $\mathrm{n}^{\circ}$ 106, vol.32, décembre 2005, pp. 501-503.

42. François Egil, «Les éléphants de papier. Réflexions impies pour le $5^{\mathrm{e}}$ anniversaire des Objectifs de développement du Millénaire ", Politique Africaine, n 99, octobre 2005, pp. 97-115.

43. Constat fait dès 1990 par James Ferguson, The Anti-Politics Machine. Development, Depolitization and Bureaucratic Power in Lesotho, Cambridge, Cambridge University Press, 1990.

44. F. Egil, art. cit, p. 108.

45. Ainsi, en dépit du boom démographique et des restrictions budgétaires des années 1980-1990, les inscriptions en primaire sautent de $43 \%$ en 1960 à $77 \%$ en 1997 alors que l'espérance de vie passe de 40 ans à 52 ans entre 1960 et 1990 avant de reculer avec l'épidémie de sida dans les années 1990-2000. Cooper, op. cit, pp. 107-110.

46. J.-F. Bayart, Le gouvernement du monde. Une critique politique de la globalisation, Paris, Fayard, 2004.

47. J.-F. Bayart, op. cit, pp. 69-82 ; Béatrice Hibou (dir.), La privatisation des États, Paris, Karthala, 1999 ; Achille Mbembe, «Du gouvernement privé indirect», in De la postcolonie. Essai sur l'imagination politique dans l'Afrique contemporaine, Paris, Karthala, 2005, pp. 95-139. Du côté des historiens, voir par exemple, l'ouvrage pionner de Catherine Coquery-Vidrovicth, Le Congo (AEF) au temps des grandes compagnies concessionnaires, 1898-1930, Paris, Mouton, 1972. 


\section{RÉSUMÉS}

Champ d'action diplomatique ou militaire des puissances occidentales (Grande-Bretagne, France, États-Unis), le continent africain se trouve aujourd'hui au cœur de certains impératifs mondiaux (intégration de la sécurité au développement, lutte contre la pauvreté). De nouveaux acteurs internationaux se sont affirmés ces quinze dernières années : les deux puissances régionales au sud du Sahara (l'Afrique du Sud et le Nigeria) et les deux principales organisations continentales (l'Union Africaine et le Nouveau partenariat pour le développement de l'Afrique, NEPAD) ont acquis une légitimité internationale les autorisant à intervenir plus directement qu'auparavant dans les affaires africaines. Il n'en demeure pas moins que la résilience d'un autoritarisme hérité des périodes coloniales et postcoloniales persiste dans bien des pays, même si un esprit de résistance démocratique s'est diffusé depuis les années 1990. Les États d'Afrique fonctionnent, même s'ils sont très fragiles dans la plupart des cas. Les défis à affronter restent considérables, tandis que leurs marges de manœuvre demeurent aujourd'hui, sauf exception, très réduites.

Africa has recently attracted a new wave of diplomatic and military action from major western countries (France, United Kingdom, and the United States). At the same time the continent is at the centre of new international imperatives such as poverty reduction and a more comprehensive integration of security needs into the development agenda. New international players have however emerged in the last fifteen years. The two sub-Saharan regional powers (South Africa and Nigeria) and the two principal continental organisations (the African Union and the New Partnership for Africa's Development, NEPAD) have recently acquired a new international legitimacy which authorises them to intervene more directly into African affairs. Nevertheless the authoritarianism inherited from the colonial and postcolonial times remains strong in many countries even though a spirit of democratic resistance appears to have become widespread since the 1990s. The majority of African States function, even though they are very fragile. The challenges they face are significant whilst their room for manoeuvre is, generally, extremely limited.

\section{INDEX}

Keywords : News players, conflicts, democratisation, mobilities

Mots-clés : Nouveaux acteurs, conflits, démocratisation, mobilités

Index géographique : Afrique

\section{AUTEUR}

\section{LAURENT FOURCHARD}

Agrégé et docteur en histoire de l'université de Paris 7, Laurent Fourchard est chercheur à la Fondation nationale des sciences politiques (Centre d'étude d'Afrique noire de Bordeaux). Ancien directeur de l'Institut français de recherche en Afrique (IFRA) à Ibadan au Nigeria, il a travaillé sur les dynamiques urbaines, sur la criminalité et l'État, et sur les réseaux religieux en Afrique de l'Ouest. Il a récemment co-dirigé Sécurité, crime et ségrégation dans les villes d'Afrique de l'Ouest et Entreprises religieuses transnationales en Afrique de l'Ouest, parus chez Karthala respectivement en 2003 et 2005. Il coordonne depuis 2006 un Groupement de recherche international 
(GDRI) du CNRS sur « Gouverner les villes d'Afrique : lois, institutions locales et identités urbaines depuis $1945 »$.

1.fourchard@sciencespobordeaux.fr 